Published in final edited form as:

J Infect. 2017 October ; 75(4): 370-373. doi:10.1016/j.jinf.2017.05.014.

\title{
Bronchoalveolar lavage triacetylfusarinine C (TAFC) determination for diagnosis of invasive pulmonary aspergillosis in patients with hematological malignancies
}

\author{
Thomas Orascha\#, \\ Division of Molecular Biology, Innsbruck Medical University, Innsbruck, Austria \\ Juergen Prattesa\#, \\ Section of Infectious Diseases and Tropical Medicine, Medical University of Graz, Graz, Austria; \\ CBmed Center for Biomarker Research in Medicine, Graz, Austria \\ Klaus Faserl, \\ Division of Clinical Biochemistry, Innsbruck Medical University, Innsbruck, Austria \\ Susanne Eigl, \\ Division of Pulmonology, Medical University of Graz, Graz, Austria \\ Wiebke Düttmann, \\ Section of Infectious Diseases and Tropical Medicine, Medical University of Graz, Graz, Austria \\ Herbert Lindner, \\ Division of Clinical Biochemistry, Innsbruck Medical University, Innsbruck, Austria \\ Hubertus Haas ${ }^{*}$, and \\ Division of Molecular Biology, Innsbruck Medical University, Innsbruck, Austria \\ Martin Hoenigl $^{\star *}$ \\ Section of Infectious Diseases and Tropical Medicine, Medical University of Graz, Graz, Austria; \\ CBmed Center for Biomarker Research in Medicine, Graz, Austria; Division of Pulmonology, \\ Medical University of Graz, Graz, Austria; Division of Infectious Diseases, Department of \\ Medicine, University of California San Diego, San Diego, CA, USA \\ \# These authors contributed equally to this work.
}

\footnotetext{
Wriginal data of this manuscript have been presented at ID Week 2016, New Orleans, USA (poster presentation number 1558; recipient of ID Week Trainee Travel Grant).

"Corresponding author. Division of Molecular Biology, Biocenter, Medical University of Innsbruck, Innrain 80-82, A-6020 Innsbruck, Austria. hubertus.haas@i-med.ac.at. " Corresponding author. Section of Infectious Diseases and Tropical Medicine, Division of Pulmonology, Department of Internal Medicine, Medical University of Graz, Auebruggerplatz 15, A-8036 Graz, Austria. martin.hoenigl@medunigraz.at.

Conflicts of interest

M. Hoenigl received research grants from Merck, Pfizer and Gilead; served on the speakers' bureau of Pfizer, Gilead, Astellas, Basilea and Merck and received travel grants from Astellas, Merck, Gilead and Pfizer.

J. Prattes received travel grant from Pfizer and consulting fee from Gilead.

All other authors no conflict.
} 


\section{Keywords}

Siderophores; Galactomannan; Lateral flow device; Antimould treatment; Antifungal prophylaxis

\section{Dear Editor,}

We read with interest the paper by Fortun and colleagues1 who found that galactomannan (GM) testing from bronchoalveolar lavage fluid (BALF) is a promising method for detecting invasive pulmonary aspergillosis (IPA) in patients at risk. As an important limitation, sensitivity of BALF GM may decrease in case of administration of anti-mould prophylaxis or empirical therapy,2,3 which are commonly used in high-risk patients with underlying hematological malignancies to reduce the incidence of IPA and improve survival. To increase sensitivity, recent studies suggest to combine BALF GM with other diagnostic tests, such as the Aspergillus specific Lateral Flow Device Test (LFD), which is yet not commercially available, or BALF PCR, which lacks standardization.3-5 Improved diagnostic markers that can be used as combination partners with BALF GM are therefore needed.

Triacetylfusarinine $\mathrm{C}$ (TAFC) is one of two different secreted siderophores (i.e. lowmolecular mass, ferric iron-specific chelators) produced by Aspergillus fumigatus to mediate iron acquisition from the host during infection.6,7 TAFC is a fungal specific molecule that is also produced by a limited number of other moulds (e.g. Aspergillus nidulans and Fusarium graminearum), but not yeasts, and animal models have revealed promising results for TAFCmediated diagnosis of IPA.7,8 Here we evaluated in patients with hematological malignancies whether sensitivity of BALF GM can be increased by combination with TAFC.

A total of 45 BALF samples obtained from 45 patients with underlying hematological malignancies (15 patients with proven or probable IA and 30 controls with no IPA who were each matched 2:1 by age and underlying diseases) were included in this analysis. IPA was classified according to the revised EORTC/MSG criteria with one modification: exclusion of beta-D-Glucan as mycological criterion.9 BALF samples were obtained between July 2012 and August 2015 at the Medical University Hospital Graz, Austria. GM (Platelia Aspergillus Ag ELISA; Bio-Rad Laboratories, Munich, Germany) and LFD (OLM Diagnostics, Newcastle upon Tyne, UK) 10 were performed prospectively in all samples, as described previously. 10 Samples were thereafter stored at $-70{ }^{\circ} \mathrm{C}$ and shipped in 2015 on dry ice to the Innsbruck Medical University for retrospective mass spectrometry-mediated TAFC determination.7 Investigators in Innsbruck were blinded towards IPA classification of the samples. For BALF GM we evaluated two different cut-offs for determining positivity: $>0.5$ optical density index (ODI), and $>1.0 \mathrm{ODI}$, which has recently been recommended by the FDA in its Guidance on Qualification of Biomarkers. Receiver operating characteristic (ROC) curves analyses were performed, and area under the curve (AUC) values are presented including 95\% confidence intervals (CI) for TAFC. The optimal cut-off for discriminating patients with and without IPA was calculated by using the Youdens index. The study adhered to Declaration of Helsinki, 1996, Good Clinical Practice, and was approved by the local ethics committee, Medical University Graz, Austria (EC-number 23- 
343 ex 10/11). Statistical analysis was performed using SPSS, version 23 (SPSS Inc., Chicago, IL, USA). Negative predictive value (NPV), positive predictive value (PPV), sensitivity and specificity for single biomarkers and combinations were calculated. A pvalue of $<0.05$ was considered as statistically significant.

A total of 44 samples from 15 patients with probable $(n=14)$ or proven $(n=1)$ IPA and 29 controls without evidence of IPA were included in the final analysis (one sample had to be excluded because TAFC measurement failed). Demographic characteristics and underlying diseases of the study population are displayed in Table 1. A total of 11/15 (73\%) of patients with probable/proven IPA were receiving mould-active antifungal prophylaxis/therapy at the time of the BALF procedure. AUC for TAFC for differentiating probable/proven from no IPA was 0.601 (0.425-0.777, n.s.), and with Youdes Index we determined an optimal cut-off of $>1 \mathrm{ng} / \mathrm{ml}$. Performances of TAFC, GM and LFD as well as combinations are depicted in Table 2. Range of TAFC levels that were considered true positives (i.e. measured in patients with IPA) was $1.4-6.2 \mathrm{ng} / \mathrm{ml}$, range of false positive TAFC levels was $1.2-3.3 \mathrm{ng} / \mathrm{ml}$. While sensitivity of BALF GM as a single test was $73 \%$ with the 0.5 ODI cut-off, and $53 \%$ when using the 1.0 ODI cut-off, sensitivities could be markedly increased when combining GM with either TAFC or LFD. The combination of either TAFC and/or GM resulting positive exhibited sensitivities of $87 \%$ (with 0.5 ODI GM cut-off) and $73 \%$ (with 1.0 ODI cut-off) with similar results observed for the combination LFD and/or GM. In two IPA patients with negative BALF GM $(<0.5 \mathrm{ODI})$, both TAFC and LFD resulted positive (both samples resulted also positive with Aspergillus specific BALF PCR4,5 which was performed only in a subset of samples), indicating the additional diagnostic value of both, TAFC and the LFD. While only four BALF samples resulted positive for both TAFC and GM, all these samples originated from patients with probable/proven IPA (100\% specificity and PPV).

IPA is associated with high morbidity and mortality in hematological malignancy patients, and early detection is essential for optimal therapeutic success. While diagnostic efforts have been perpetually improved, the optimal use of the available diagnostic repertoire is still a matter of debate. This is true in particular in patients receiving mould active prophylaxis which has been shown to reduce sensitivity of GM and other diagnostic tests for IPA.2 In the present study we evaluated in patients with hematological malignancies (of which $73 \%$ received antimould prophylaxis/empirical treatment at the time of bronchoscopy), whether sensitivity of BALF GM can be increased by combination with TAFC, a mould specific siderophore, or the LFD. Our main finding was that sensitivities of BALF GM were increased from $53 \%$ to $73 \%$ (1.0 ODI GM cut-off), and from $73 \%$ to $87 \%$ (0.5 ODI GM cutoff), when combining with TAFC, with comparable results observed for the GM LFD combination. The latter is in line with previous studies evaluating BALF GM LFD combination.3,5 Limitations of our study include that all but one IPA case were probable diagnoses, which introduces a bias towards overestimation of BALF GM performance. Studies in cases with proven IPA are needed to overcome this issue.

In conclusion, the combination of TAFC and GM in BALF was promising for confirming (PPV of $100 \%$ when both resulted positive) and ruling out IPA (NPV of $92 \%$ when both resulted negative). More studies regarding this new and promising biomarker need to be 
performed to define the optimal cut-off and investigate the diagnostic performance in other specimen and larger patient cohorts.

\section{Funding}

This work was supported by the Austrian science funds doctoral programme "host response in opportunistic infections" (HOROS, W1253) and funds from the Oesterreichische Nationalbank (Anniversary Fund, project number 15346). The funders had no role in study design, data collection, analysis, interpretation, decision to publish, in the writing of the manuscript, and in the decision to submit the manuscript for publication.

\section{References}

1. Fortun J, Martin-Davila P, Gomez Garcia de la Pedrosa E, Silva JT, Garcia-Rodriguez J, Benito D, et al. Galactomannan in bronchoalveolar lavage fluid for diagnosis of invasive aspergillosis in nonhematological patients. J Infect. 2016 Jun; 72(6):738-44. [PubMed: 27025205]

2. Eigl S, Prattes J, Reinwald M, Thornton CR, Reischies F, Spiess B, et al. Influence of mould-active antifungal treatment on the performance of the Aspergillus-specific bronchoalveolar lavage fluid lateral-flow device test. Int J Antimicrob Agents. 2015; 46(4):401-5. [PubMed: 26187363]

3. Prattes J, Lackner M, Eigl S, Reischies F, Raggam RB, Koidl C, et al. Diagnostic accuracy of the Aspergillus-specific bronchoalveolar lavage lateral-flow assay in haematological malignancy patients. Mycoses. 2015 Aug; 58(8):461-9. [PubMed: 26103209]

4. Eigl S, Hoenigl M, Spiess B, Heldt S, Prattes J, Neumeister P, et al. Galactomannan testing and Aspergillus PCR in same-day bronchoalveolar lavage and blood samples for diagnosis of invasive aspergillosis. Med Mycol. 2016 Oct 14.

5. Hoenigl M, Prattes J, Spiess B, Wagner J, Prueller F, Raggam RB, et al. Performance of galactomannan, beta-d-glucan, Aspergillus lateral-flow device, conventional culture, and PCR tests with bronchoalveolar lavage fluid for diagnosis of invasive pulmonary aspergillosis. J Clin Microbiol. 2014 Jun; 52(6):2039-45. [PubMed: 24671798]

6. Haas H. Iron - a key nexus in the virulence of Aspergillus fumigatus. Front Microbiol. 2012 Feb 6.3:28. [PubMed: 22347220]

7. Carroll CS, Amankwa LN, Pinto LJ, Fuller JD, Moore MM. Detection of a serum siderophore by LC-MS/MS as a potential biomarker of invasive Aspergillosis. PLoS One. $2016 \mathrm{Mar}$ 14.11(3):e0151260. [PubMed: 26974544]

8. Haas H, Petrik M, Decristoforo C. An iron-mimicking, Trojan horse-entering fungie-has the time come for molecular imaging of fungal infections? PLoS Pathog. 2015 Jan 29.11(1):e1004568. [PubMed: 25634225]

9. De Pauw B, Walsh TJ, Donnelly JP, Stevens DA, Edwards JE, Calandra T, et al. Revised definitions of invasive fungal disease from the European Organization for Research and Treatment of Cancer/ Invasive Fungal Infections Cooperative Group and the National Institute of Allergy and Infectious Diseases Mycoses Study Group (EORTC/MSG) Consensus Group. Clin Infect Dis. 2008 Jun 15; 46(12):1813-21. [PubMed: 18462102]

10. Hoenigl M, Koidl C, Duettmann W, Seeber K, Wagner J, Buzina W, et al. Bronchoalveolar lavage lateral-flow device test for invasive pulmonary aspergillosis diagnosis in haematological malignancy and solid organ transplant patients. J Infect. 2012 Dec; 65(6):588-91. [PubMed: 23078908] 


\section{Table 1}

Demographic data and underlying diseases of the study population.

\begin{tabular}{lll}
\hline & Probable or proven IPA & No evidence for IPA \\
\hline Number & 15 & 29 \\
Female (n, \%) & $9(60)$ & $17(59)$ \\
Age, years (median, IQR) & $60(56-65)$ & $61(53-69)$ \\
Underlying diseases (n, \%) & & \\
Acute myeloid leukemia & $8(53)$ & $16(55)$ \\
Multiple myeloma & $3(20)$ & $6(21)$ \\
Acute lymphocytic leukemia & $2(13)$ & 27 \\
Chronic lymphocytic leukemia & $1(7)$ & $2(7)$ \\
Non-hodgkin lymphoma & $1(7)$ & $2(7)$ \\
Myelodysplastic syndrome & - & $1(3)$ \\
\hline
\end{tabular}




\section{Table 2}

Diagnostic performance of Triacetylfusarinine C (TAFC), Aspergillus specific Lateral Flow Device Test (LFD), galacto-mannan enzyme immunoassay (GM) and conventional microbiological culture (culture) in bronchoalveolar lavage fluid (BALF) samples as single tests or in distinct combinations.

\begin{tabular}{lllll}
\hline Biomarkers/tests and combinations & Sensitivity & Specificity & PPV & NPV \\
\hline TAFC & $40 \%(6 / 15)$ & $79 \%(23 / 29)$ & $50 \%(6 / 12)$ & $72 \%(23 / 32)$ \\
LFD & $47 \%(7 / 15)$ & $79 \%(23 / 29)$ & $54 \%(7 / 13)$ & $74 \%(23 / 31)$ \\
GM (0.5 ODI) ${ }^{a}$ & $73 \%(11 / 15)$ & $97 \%(28 / 29)$ & $92 \%(11 / 12)$ & $88 \%(28 / 32)$ \\
GM (1.0 ODI) ${ }^{a}$ & $53 \%(8 / 15)$ & $97 \%(28 / 29)$ & $89 \%(8 / 9)$ & $80 \%(28 / 35)$ \\
Fungal culture ${ }^{a}$ & $13 \%(2 / 15)$ & $100 \%(29 / 29)$ & $100 \%(2 / 2)$ & $69 \%(29 / 42)$ \\
TAFC and/or GM (0.5 ODI) & $87 \%(13 / 15)$ & $76 \%(22 / 29)$ & $65 \%(13 / 20)$ & $92 \%(22 / 24)$ \\
TAFC and/or GM (1.0 ODI) & $73 \%(11 / 15)$ & $76 \%(22 / 29)$ & $61 \%(11 / 18)$ & $85 \%(22 / 26)$ \\
LFD and/or GM (0.5 ODI) & $93 \%(14 / 15)$ & $76 \%(22 / 29)$ & $67 \%(14 / 21)$ & $96 \%(22 / 23)$ \\
LFD and/or GM (1.0 ODI) & $73 \%(11 / 15)$ & $76 \%(22 / 29)$ & $61 \%(11 / 18)$ & $85 \%(22 / 26)$ \\
TAFC and/or LFD & $67 \%(10 / 15)$ & $62 \%(18 / 29)$ & $48 \%(10 / 21)$ & $78 \%(18 / 23)$ \\
TAFC and/or LFD and/or GM (1.0 ODI) & $80 \%(12 / 15)$ & $59 \%(17 / 29)$ & $50 \%(12 / 24)$ & $75 \%(17 / 20)$ \\
\hline
\end{tabular}

${ }^{a}$ In contrast to TAFC and LFD, GM and culture are part of the mycological criteria of the EORTC/MSG criteria and were therefore used for classification of IPA leading to potential overestimation of diagnostic performance. 